\section{Hämoglobin A1}

K. J. Lackner ${ }^{1}$ und D. Peetz ${ }^{2}$

${ }^{1}$ Institut für Klinische Chemie und Laboratoriumsmedizin, Universitätsmedizin Mainz, Mainz, Deutschland

${ }^{2}$ Institut für Labormedizin, Helios Klinikum Berlin-Buch, Berlin, Deutschland

$\operatorname{Synonym(e)~} \mathrm{HbA}_{1}$

Englischer Begriff hemoglobin A1

Definition Am N-terminalen Valin der $\beta$-Globinkette glykiertes Hämoglobin.
Beschreibung $\mathrm{HbA}_{1}$ ist ein Gemisch aus $\mathrm{HbA}$, das mit unterschiedlichen Kohlenhydraten glykiert ist: $\mathrm{HbA}_{1 \mathrm{a} 1}$ Fruktose-1,6-Diphosphat, $\quad \mathrm{HbA}_{1 \mathrm{a} 2}$-Glukose-6-Phosphat, $\mathrm{HbA}_{1 \mathrm{~b}}$-unbekanntes Kohlenhydrat, $\mathrm{HbA}_{1 \mathrm{c}}$-Glukose. Für die Diagnostik ist $\mathrm{HbA}_{1}$ obsolet. Es sollte im Hinblick auf die Standardisierung und Aussagekraft angestrebt werden, nur $\mathrm{HbA}_{1 \mathrm{c}}$ spezifisch zu messen, das auch als einziges glykiertes Hämoglobin in den diversen Leitlinien geführt wird.

\section{Literatur}

John WG (2003) Haemoglobin A1c: analysis and standardisation. Clin Chem Lab Med 41:1199-1212 